\title{
Desafios encontrados pela medicina integrativa no âmbito do Sistema Único de Saúde (SUS): uma revisão integrativa
}

\author{
Challenges encountered by integrative medicine within the scope of the Unified Health \\ System (SUS): an integrative review
Retos de la medicina integrativa en el ámbito del Sistema Único de Salud (SUS): una revisión integradora

Gustavo Gregorio Dias Firmiano1, Felipe Ribeiro Dutra Palhares Ferreira', Gustavo de Godoi

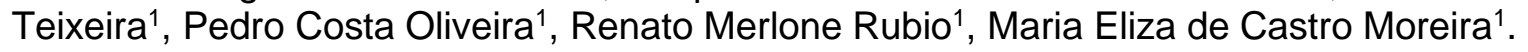

\section{RESUMO}

Objetivo: Conhecer e traçar o perfil de implementação da Medicina Integrativa no Brasil, bem como as adversidades enfrentadas pelas Práticas Integrativas e Complementares dentro do Sistema Único de Saúde. Métodos: $O$ estudo se trata de uma revisão integrativa de literatura com dados retirados nas bases eletrônicas de dados Medical Literature Analysis and Retrieval System Online (MEDLINE) via PubMED, Biblioteca Virtual em Saúde do Ministério da Saúde (BVS) via LILACS e Scientific Electronic Library Online (SciELO), nas quais foram selecionados artigos originais publicados entre os anos de 2016 a 2021 . Resultados: Tais práticas estão cada vez mais acessíveis, no entanto, não estão difunfidas entre milhões de pessoas. Esse impedimento se dá graças ao desalinhamento entre politicas públicas e privadas, além de uma baixa implantação das PICs pelo cenário nacional. Considerações finais: A carência da medicina alternativa no Brasil é um reflexo da falta de apoio governamental e preparo administrativo das unidades de saúde, mescladas a um plano de ensino superior que não promove essas práticas no currículo dos futuros profissionais da área.

Palavras-chave: Medicina integrativa nacional, Práticas integrativas complementares (PIC), Terapias complementares.

\begin{abstract}
Objective: To know and profile the implementation of Integrative Medicine in Brazil, as well as the adversities faced by Integrative and Complementary Practices within the Unified Health System. Methods: The study is an integrative literature review with data retrieved from the electronic databases Medical Literature Analysis and Retrieval System Online (MEDLINE) via PubMED, Virtual Health Library of the Ministry of Health (VHL) via LILACS and Scientific Electronic Library Online (SciELO), in which original articles published between the years 2016 and 2021 were selected. Results: Such practices are increasingly accessible, however, they are not diffused among millions of people. This impediment is due to the misalignment between public and private policies, in addition to a low implementation of PICs throughout the national scenario. Final considerations: The lack of alternative medicine in Brazil is a reflection of the lack of governmental support and administrative preparation of health care units, mixed with a higher education plan that does not promote these practices in the curriculum of future professionals.
\end{abstract}

Key words: National integrative medicine, Complementary integrative practices (PIC), Complementary therapies.

\section{RESUMEN}

Objetivo: Conocer y perfilar el perfil de la implantación de la Medicina Integrativa en Brasil, así como las adversidades a las que se enfrentan las Prácticas Integrativas y Complementarias dentro del Sistema Único de Salud. Métodos: El estudio es una revisión bibliográfica integradora con datos tomados de las bases de datos electrónicas Medical Literature Analysis and Retrieval System Online (MEDLINE) a través de PubMED,

${ }^{1}$ Faculdade Dinâmica do Vale do Piranga (FADIP), Ponte Nova - MG. *E-mail: Gust.dias14@gmail.com 
Biblioteca Virtual de Salud del Ministerio de Salud (BVS) a través de LILACS y Scientific Electronic Library Online (SciELO), en las que se seleccionaron artículos originales publicados entre los años 2016 y 2021. Resultados: Estas prácticas son cada vez más accesibles, pero no están difundidas entre millones de personas. Este impedimento se debe a la desalineación entre las políticas públicas y privadas, además de una escasa implantación de los PIC en el ámbito nacional. Consideraciones finales: La falta de medicina alternativa en Brasil es un reflejo de la falta de apoyo gubernamental y preparación administrativa de las unidades de salud, mezclado con un plan de educación superior que no promueve estas prácticas en el currículo de los futuros profesionales del área.

Palabras clave: Medicina integrativa nacional, Prácticas complementarias integrativas (PIC), Terapias complementarias.

\section{INTRODUÇÃO}

A Medicina Integrativa vem sendo discutida desde a VIII Conferência Nacional de Saúde em 1986 (SILVA GK, et al., 2020). Porém essa prática foi introduzida no Brasil de forma mais consistente apenas em 2006 pelo Ministério da Saúde (MS). Já em 2017, foi ampliada e novos tipos de terapias integrativas e complementares foram indexadas à portaria número 971/2006 (SILVEIRA RP e ROCHA CM, 2020).

Essa portaria publicada em 2006, que aprovou a Política Nacional de Práticas Integrativas e Complementares (PNPIC), trouxe ao Sistema Único de Saúde (SUS) diferentes métodos terapêuticos: homeopatia, medicina tradicional chinesa, acupuntura, fitoterapia, medicina antroposófica, termalismo ou crenoterapia, arteterapia, ayurveda, biodança, dança circular, ioga, meditação, musicoterapia, naturopatia, osteopatia, quiropraxia, reflexoterapia, reiki, shantala e terapia comunitária (SILVEIRA RP e ROCHA CM, 2020).

Essas Práticas Integrativas e Complementares (PICs) têm como objetivo promover a saúde estimulando o vínculo entre profissionais de saúde e pacientes, interação humana com o meio ambiente e sociedade, prevenir agravos de condições crônicas, possibilitar uma recuperação saudável, e se necessário, a utilização de tecnologias para maior eficiência (RUELA LO, et al., 2019).

As PIC's podem ser classificadas de acordo com sua conjuntura com a biomedicina. Quando utilizadas mutuamente são chamadas de complementares. Assim, quando a é colocada no lugar de uma prática biomédica são consideradas alternativas e quando são usadas conjuntamente e baseadas em avaliações científicas de segurança e eficácia de boa qualidade, chamadas de integrativas (BARROS LCN, et al., 2020).

As PICs possuem uma vasta gama de segmentos, como: Sistemas médicos alternativos (homeopatia, medicina ayurvédica, e outras), intervenções mente-corpo (meditações, yoga, orações), terapias biológicas (baseados em produtos naturais não reconhecidos cientificamente), métodos de manipulação corporal e baseados no corpo (massagens, exercícios), e terapias energéticas, como Reiki, Tai chi chuan, dentre outras (BARROS LCN, et al., 2020; FAQUETI A e TESSER CD, 2018).

Entretanto, essas práticas passaram a ser um estigma, visto que, para inseri-las na atenção primária necessitaria de alguns encargos, como viabilizar os profissionais, monitorá-los e avaliá-los, comprar insumos (agulhas para acupuntura, medicamentos homeopáticos, fitoterápicos, entre outros) e realizar análises periódicas no intuito de preservar a qualidade do serviço (HABIMORAD PH, et al., 2020).

Foi assim que em 2011 foi criado o Programa Nacional de Melhoria do Acesso e da Qualidade da Atenção Básica (PMAQ-AB) com o propósito de melhorar a qualidade dos serviços por meio de estratégias do MS junto ao Departamento de Atenção Básica (DAB), e consequentemente trazer avanços as PICs (VIEIRA IC, et al., 2018).

Desse modo, a procura pelas PICs aumentou e mais médicos passaram a orientar seus pacientes a respeito dessa "nova" modalidade, porém percebeu-se que a população predominante na busca pelos serviços eram mulheres, brancas, católicas, com escolaridade incompleta e faixa etária variada. Assim, notase a desproporção do sexo feminino e masculino na saúde pública e a desigualdade social e racial em toda 
a Atenção Básica à Saúde (ABS), esbarrando com mais obstáculos na integração da Medicina Complementar no SUS, visto que, as PIC's tem como objetivo promover tanto a saúde da mulher quando do homem (VIEIRA IC, et al., 2018).

Acrescenta-se também a esse emaranhado de desafios enfrentados pelas PICs, a falta de interesse das escolas em lecionar sobre as Práticas Integrativas e Complementares, dessa forma, privam os alunos do conhecimento sobre a Medicina Integrativa (ALMEIDA JR, et al., 2016).

A incorporação dessas práticas ao currículo acadêmico acrescentaria e fortaleceria os princípios do SUS, reduziria a utilização de medicamentos, aumentaria a adesão ao tratamento, devido aos variados estilos, e a valorização cultural seria evidente, pois, um dos princípios é o olhar holístico com respeito a crenças, valores e individualidades (SANTOS LL, et al., 2018; DALMOLIN IS e HEIDEMANN IT, 2020).

Por fim, o impacto do PNPIC vem sendo efetivo e atinge campos econômicos, técnicos, sociopolíticos, educacionais e acadêmicos. No entanto, as PICs ainda devem ser divulgadas para continuar progredindo e expandindo, ancorando-as aos princípios integrativos do SUS, acolhimento, interação e acessibilidade, no intuito de melhorar a qualidade de vida dos pacientes (BARROS LC, et al., 2020).

Assim, o objetivo do tarbalho foi conhecer e traçar o perfil de implementação da Medicina Integrativa no Brasil, bem como as adversidades enfrentadas pelas PIC's dentro do SUS.

\section{MÉTODOS}

Trata-se de uma revisão integrativa, a qual traz a importância da Medicina Integrativa no mundo contemporâneo. As buscas foram realizadas no mês de março do ano 2021, por meio de consultas às bases de dados, Medical Literature Analysis and Retrieval System Online (MEDLINE) via PubMED, Biblioteca Virtual em Saúde do Ministério da Saúde (BVS) via LILACS, e Scientific Electronic Library Online (SciELO), no período de 2016 a 2021.

Os descritores empregados na pesquisa foram adquiridos através dos Descritores em Ciências da Saúde (DeCS) ou do Medical Subject Headings (MeSH). Em inglês, foram: Integrative Medicine, Complementary integrative practices (PIC), e Complementary Therapies, utilizou-se o termo "AND", cruzando sistematicamente o descritor principal (primeiro) com auxiliar (segundo). Na plataforma MEDLINE, aplicou-se primeiro, em inglês, para analisar a evolução das publicações nos últimos 70 anos.

Foram excluídas da pesquisa de meta-análises, artigos de revisão, cartas, comentários, e artigos duplicados. Foram incluídos artigos, estudos observacionais, pesquisas qualitativas, livros e documentos, constatados nos últimos cinco anos que contribuíram para a elaboração desta revisão.

\section{RESULTADOS}

Na base de dados BVS via LILACS obteve-se 32, logo, na plataforma MEDLINE via PubMED 34 artigos, e na SciELO a busca dos descritores foi com seu correspondente em português (Medicina Integrativa Nacional e Terapias Complementares), atingindo a soma de 12 artigos. Totalizando 78 artigos, sendo que 7 foram retirados por serem duplicados.

Dos 71 artigos que restaram, todos foram analisados primeiramente pelos títulos. Com isso, 15 foram eliminados devido a não correspondência ao tema proposto. Foi realizada a leitura dos resumos de todos os artigos selecionados, 56, concluídos que 23 desses não indagaram o objetivo deste estudo. Por fim, restaram 33 artigos, que foram lidos na íntegra, sendo que 15 foram excluídos, pois fugiam do tema proposto (Figura 1). 
Figura 1 - Fluxograma da seleção dos artigos.

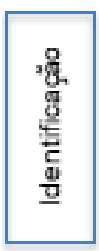

Referências identificadas através das

buscas nas bases eletrônicas ( $n=79$ )

Referências identificadas por busca manual em outras fontes $(n=0)$

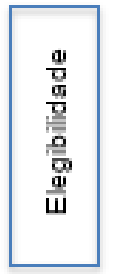

Artigos completos analisados $(n=42)$

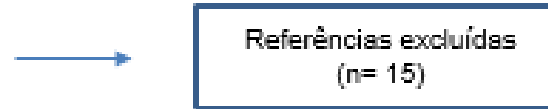

Referências selecionadas $(n=57)$ $(n=15)$

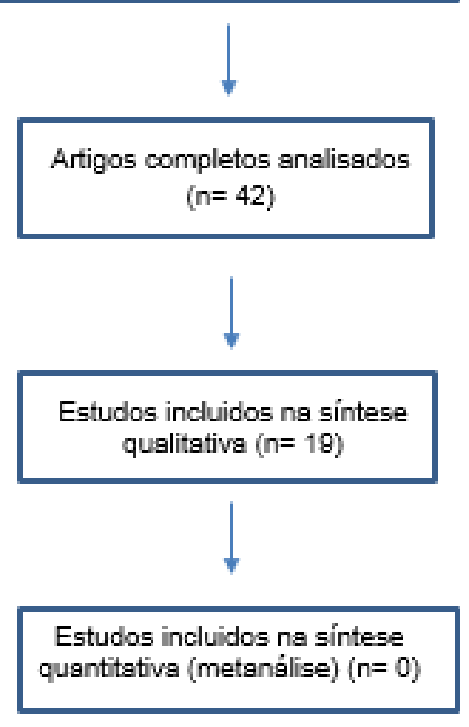

Fonte: Firmiano GGD, et al., 2021.

No Quadro 1 se encontra a descrição dos 18 artigos selecionados, com autor, data da publicação e a abordagem principal de cada trabalho. Para evidenciar o crescente interesse do assunto, buscamos o termo Medicina Integrativa Nacional na plataforma MEDLINE via PubMed. Foram identificadas 47.795 publicações. 
Quadro 1 - Artigos incluído na revisão integrativa (MEDLINE - PubMED; BVS - LILACS; SciELO).

\begin{tabular}{|c|c|c|}
\hline Autor (Ano) & Objetivo & Conclusão \\
\hline Losso LN e Freitas SF (2017) & $\begin{array}{l}\text { Aplicar um modelo de avaliação do grau de implantação } \\
\text { das Práticas Integrativas e Complementares (PIC) na } \\
\text { atenção básica nos municípios de Santa Catarina (SC). }\end{array}$ & $\begin{array}{l}\text { As variáveis obtidas do pmaq-ab não permitiram estabelecer } \\
\text { relações entre processo e estrutura das pic identificadas. }\end{array}$ \\
\hline Vieira IC, et al. (2018) & $\begin{array}{l}\text { Identificar a demanda e a proporção de uso das Práticas } \\
\text { Integrativas e Complementares (PIC) pela população } \\
\text { residente em área de abrangência de Unidade Básica de } \\
\text { Saúde (UBS). }\end{array}$ & $\begin{array}{l}\text { Existe uma demanda populacional pelas PIC que ainda não é } \\
\text { plenamente atendida pelo sistema de saúde devido à falta de } \\
\text { informação. }\end{array}$ \\
\hline Santos LL, et al. (2018) & $\begin{array}{l}\text { Investigar o interesse, o conhecimento e o uso das } \\
\text { Práticas Integrativas e Complementares (PIC) pelos } \\
\text { estudantes de medicina. }\end{array}$ & $\begin{array}{l}\text { Há necessidade da inclusão da PIC no currículo acadêmico dos } \\
\text { alunos de medicina, a fim de ampliar a formação do aluno como } \\
\text { médico generalista. }\end{array}$ \\
\hline Silveira RP e Rocha CM (2020) & $\begin{array}{l}\text { Conhecer como foram constituídos alguns dos regimes de } \\
\text { verdade sobre as Práticas Integrativas e Complementares } \\
\text { em Saúde (PICS) nas políticas públicas de saúde, } \\
\text { especialmente na Política Nacional de Práticas } \\
\text { Integrativas e Complementares (PNPIC). }\end{array}$ & $\begin{array}{l}\text { Foi concluído que as PICS, futuramente, serão mais exploradas } \\
\text { devido ao projeto de governo neoliberal proposto pela sociedade. } \\
\text { Portanto, em nossa atualidade, não somos privados apenas dos } \\
\text { nossos direitos sociais, somos privados também do acesso a saúde } \\
\text { universal gratuita. Sendo, por fim, direcionados a um viés } \\
\text { consumista de práticas de saúde alternativa. }\end{array}$ \\
\hline Dalmolin IS e heidemann IT (2020) & $\begin{array}{l}\text { Compreender a utilização de práticas integrativas e } \\
\text { complementares como ação de promoção da saúde. }\end{array}$ & $\begin{array}{l}\text { As práticas integrativas e complementares são recursos para a } \\
\text { promoção da saúde, por meio da integralidade do cuidado e } \\
\text { redução do uso de medicamentos. }\end{array}$ \\
\hline Barros IC, et al. (2020) & $\begin{array}{l}\text { Compreender os sentidos atribuídos pelos gestores dos } \\
\text { serviços da região metropolitana de goiânia sobre a oferta } \\
\text { de Práticas Integrativas e Complementares (PIC) na } \\
\text { Atenção Primária à Saúde (APS). }\end{array}$ & $\begin{array}{l}\text { Há forte influência do modelo biomédico em detrimento da } \\
\text { integralidade na organização dos serviços de APS, o que implica } \\
\text { com a baixa e descontínua oferta das PIC na região metropolitana } \\
\text { de goiânia. }\end{array}$ \\
\hline Jales RD, et al. (2020) & $\begin{array}{l}\text { Identificar o conhecimento e a aplicação das práticas } \\
\text { integrativas e complementares pelos enfermeiros da } \\
\text { atenção básica. }\end{array}$ & $\begin{array}{l}\text { O conhecimento sobre a política nacional de práticas integrativas e } \\
\text { complementares ainda há limitações no sistema de saúde. }\end{array}$ \\
\hline
\end{tabular}




\begin{tabular}{|c|c|c|}
\hline Autor (Ano) & Objetivo & Conclusão \\
\hline Habimorad PH, et al. (2020) & $\begin{array}{l}\text { Analisar a produção científica sobre as Práticas } \\
\text { Integrativas e Complementares (PIC) no Sistema Único } \\
\text { de Saúde (SUS) visando compreender as potencialidades } \\
\text { e fragilidades do processo de implantação. }\end{array}$ & $\begin{array}{l}\text { Diante desse cenário, é de desafio no SUS que possamos debater } \\
\text { acerca da PNPIC. Pois, há necessidade de articulação política de } \\
\text { seus atores para seu avanço. }\end{array}$ \\
\hline Ruela LO, et al. (2019) & $\begin{array}{l}\text { Analisar a implantação, acesso e utilização dos pics no } \\
\text { Sistema Único de Saúde (SUS) após o estabelecimento } \\
\text { desta política. }\end{array}$ & $\begin{array}{l}\text { As pics são oferecidas de maneira tímida e os dados disponíveis } \\
\text { são escassos, apesar dos impactos positivos sobre os usuários e } \\
\text { os serviços que adotaram seu uso. }\end{array}$ \\
\hline Faqueti A e Tesser CD (2018) & $\begin{array}{l}\text { Analisar parcialmente os resultados de uma pesquisa que } \\
\text { teve como princípio a percepção dos usuários sobre o uso } \\
\text { institucional da Medicina Complementar e Alternativa } \\
\text { (MAC) nos centros de saúde de florianópolis. }\end{array}$ & $\begin{array}{l}\text { A necessidade de expandir e formar profissionais (biomédicos), } \\
\text { exigirá também uma ampliação do atual, inexistente, treinamento } \\
\text { acerca da MAC para estudantes e profissionais em atividade. }\end{array}$ \\
\hline Silva GK, et al. (2020) & $\begin{array}{l}\text { Analisar a construção e desenvolvimento da Política } \\
\text { Nacional de Práticas Integrativas e Complementares } \\
\text { (PNPIC) no Sistema Único de Saúde (SUS) e seus } \\
\text { antecedentes. }\end{array}$ & $\begin{array}{l}\text { A integração das pics no sus não deveria limitar-se à adição de mais } \\
\text { procedimentos advindos de racionalidades externas à biomedicina, } \\
\text { e sim contribuir para a produção de mudanças no cuidado e no } \\
\text { cotidiano dos serviços em saúde. }\end{array}$ \\
\hline Almeida JR, et al. (2016) & $\begin{array}{l}\text { Verificar o conhecimento de estudantes universitários } \\
\text { sobre medicina alternativa. }\end{array}$ & $\begin{array}{l}\text { Os estudantes universitários pouco conhecem sobre medicina } \\
\text { alternativa, práticas integrativas e complementares devido a pouca } \\
\text { influência governamental. }\end{array}$ \\
\hline Nascimento MC, et al. (2018) & $\begin{array}{l}\text { Analisar a oferta de disciplinas relacionadas à medicina } \\
\text { integrativa em seis universidades públicas do Estado do } \\
\text { Rio de Janeiro. }\end{array}$ & $\begin{array}{l}\text { Ficou compreendido que os cursos de medicina, enfermagem e } \\
\text { farmácia apresentam subáreas em saúde integrativa, porém a } \\
\text { maioria é de caráter opcional ou apenas informativo. }\end{array}$ \\
\hline Albuquerque IV, et al. (2019) & $\begin{array}{l}\text { Avaliar a implementação de práticas integrativas no plano } \\
\text { de ensino das escolas médicas no brasil. }\end{array}$ & $\begin{array}{l}\text { Foi comprovado que, mesmo após a publicação da pnpic, não } \\
\text { houve crescimento do ensino de práticas integrativas nas escolas } \\
\text { de medicina no brasil. }\end{array}$ \\
\hline Damasceno CM, et al. (2016) & $\begin{array}{l}\text { Avaliar o conhecimento de estudantes universitários a } \\
\text { respeito do tema medicina integrativa. }\end{array}$ & $\begin{array}{l}\text { Ficou evidente que o conhecimento sobre medicina integrativa é } \\
\text { baixo dentre os estudantes e que, mesmo com o apoio do ministério } \\
\text { da saúde, as universidades não implementam esse tema no plano } \\
\text { de ensino. }\end{array}$ \\
\hline
\end{tabular}




\begin{tabular}{|c|c|c|}
\hline Autor (Ano) & Objetivo & Conclusão \\
\hline Silveira MR, et al. (2011) & $\begin{array}{l}\text { Entender as possibilidades do trabalho em equipe na esf } \\
\text { e avaliar o limite da implantação da promoção a saúde. }\end{array}$ & $\begin{array}{l}\text { Ficou evidente que a promoção a saúde ainda está em comflito com } \\
\text { a sociedade e seu planejamento estratégico. Com isso, foi } \\
\text { comprovado que as abordagens interdisciplinares devem ser } \\
\text { favorecidas e contruidas através da interação e comprometimento } \\
\text { dos setores da sociedade e inclusos junto as ações estratégicas da } \\
\text { saúde. }\end{array}$ \\
\hline Cruz PL e Sampaio SF (2016) & $\begin{array}{l}\text { Revisar a literatura indexada em bases de dados, no } \\
\text { período de } 2006 \text { a } 2012 \text {. }\end{array}$ & $\begin{array}{l}\text { A necessidade de maiores investimentos em pesquisas, divulgação } \\
\text { e formação profissional na área, a fim de suprir uma demanda } \\
\text { crescente. }\end{array}$ \\
\hline Alonso SG, et al. (2019) & $\begin{array}{l}\text { Revisar os trabalhos existentes em medicina integrativa } \\
\text { do ano de } 2010 \text { a } 2019 \text {, através das plataformas ieee } \\
\text { xplore, pubmed, science direct, web of science e } \\
\text { researchgate. }\end{array}$ & $\begin{array}{l}\text { Ficou compreendido a partir da revisão que a medicina integrativa } \\
\text { é de suma importância para a resolução de várias doenças. Além } \\
\text { disso, o estudo visou mostrar como as práticas alternativas podem } \\
\text { contribuir para a prevenção e bom prognóstico frente a patologias. }\end{array}$ \\
\hline Drago LC (2018) & $\begin{array}{l}\text { Compreender e construir modelos teóricos de como os } \\
\text { profissionais de enfermagem colaboram para } \\
\text { implementação das Políticsa Nacionais de Práticas } \\
\text { Integrativas e Complementares (PNPIC) na rede } \\
\text { municipal de saúde de Florianopolis. }\end{array}$ & $\begin{array}{l}\text { Foi entendido que o trabalho multi e transdiciplinas são essenciais } \\
\text { para a articulação da promoção a saúde através da PNPIC. }\end{array}$ \\
\hline
\end{tabular}

Fonte: Firmiano GGD, et al., 2021. 


\section{DISCUSSÃO}

Após a análise de todos os estudos, observa-se dois eixos a serem discutidos: Os desafios que a Medicina Integrativa tem no SUS e as propostas governamentais e privadas destinadas à adesão das PICs na AB.

\section{Os desafios que a Medicina Integrativa tem no SUS}

A Medicina Tradicional Complementar Integrativa (MTCI), conhecida no Brasil por PICs, vem ampliando seu campo terapêutico e com isso ganhou espaço no âmbito do SUS, sobretudo, na Atenção Primária à Saúde (APS). Portanto, os desafios são iminentes, visto que, há baixa institucionalização da política, inexistência de financiamento indutor e carência de profissionais, além dos entraves com as faculdades Brasileiras que não lecionam sobre MTCI. Dessa forma, vem a inabilidade trabalhista dos médicos (ALMEIDA JR, et al., 2016; SILVA GKF, et al., 2020).

Atualmente no Brasil, a medicina integrativa ainda não faz parte de forma oficial da grade curricular dos cursos de medicina. Um estudo realizado em 2019 revelou que apenas 57 das 272 escolas médicas do Brasil abordam medicinas complementares e alternativas em sua grade curricular. Já para instituições de ensino público, em uma amostra de 209 instituições, apenas 43, que corresponde a 32\%, oferecem disciplinas relacionadas à PICS (ALBUQUERQUE LVC et al., 2019).

Dentre os cursos que foram avaliados, o maior número de disciplinas foi encontrado na enfermagem, seguido da medicina e fisioterapia. Porém, é um assunto que ganha cada vez mais prestígio e evidências científicas a respeito de sua eficiência, o que provavelmente significa que muito em breve tornar-se-á parte da grade disciplinar dos estudantes do Brasil como também no resto do mundo (DAMASCENO CMD, et al., 2016).

A formação da racionalidade médica é construída por meio de cursos de longa duração. No Brasil, essa modalidade é predominantemente oferecida em cursos de pós-graduação e em sua maioria em instituições privadas. Nos últimos anos, a graduação tem conquistado espaço no ensino da formação acerca das PICs. A perspectiva é como opção terapêutica. Os objetivos dessa formação é reduzir preconceitos ligados ao senso comum e estreitar a relação entre paciente e cuidado integral (NASCIMENTO MC et al., 2018; CRUZ PLB e SAMPAIO SF, 2016).

A Organização Mundial de Saúde (OMS) classifica a saúde não apenas como ausência de doenças, mas sim com um contexto mais amplo que inclui qualidade de vida e hábitos cotidianos. A partir daí surge o conceito de medicina integrativa, que atualmente faz parte do SUS e consequentemente, acaba por fazer parte da formação e do cotidiano tanto de acadêmicos de medicina como também dos médicos que já atuam (WORLD HEALTH ORGANIZATION (WHO), 1986).

É importante destacar que o papel dos médicos em atuação é de crucial importância para a divulgação das práticas medicinais alternativas e complementares, já que segundo estudos realizados na rede pública do estado de Santa Catarina; mais precisamente na cidade de Florianópolis, a iniciativa do uso de Medicina Alternativa e Complementar coube aos profissionais, estes que, durante suas consultas recomendavam tais práticas aos pacientes, que por sua vez, demonstraram-se muito satisfeitos com tais alternativas terapêuticas. Estes autores relataram a valorização que essa atitude por parte da equipe médica está incentivando cada vez mais a adesão às práticas alternativas e complementares no cenário nacional, apesar de todas as adversidades. (FAQUETTI A e TESSER CD, 2018)

Essas adversidades têm correlação com a limitada atuação pública. Números demonstrados pelo grupo trabalhista em 2017, onde declararam que apenas 3.018 municípios possuem alguma PIC, sendo que na APS a representatividade dessa modalidade é exclusivamente de 19\% (SILVA GKF, et al., 2020). Com isso é fácil perceber que a difusão das PICs pelo SUS é baixa, apesar da procura está em ascensão.

Tendo em vista esse aumento, pode-se perceber que a procura pela $\mathrm{MTCl}$ é pautada na área acadêmica, e consequentemente ocasiona um maior impacto nas orientações médicas e assim, justifica essa vasta busca pelas PICs no Brasil. Porém essa procura estava limitada a um corpo social, mulheres, brancas, católicas, com escolaridade incompleta, e faixa etária variada (VIEIRA IC et al., 2018). Essa limitação traz alguns 
questionamentos na implicação das PICs, visto que, seus princípios integrativos são, acolhimento, interação, acessibilidade e promoção a saúde (BARROS LCN, et al., 2020).

Outros obstáculos também são comuns, como as unidades semi-adaptadas e a organização das equipes. Devido ao fato de algumas UBSs funcionarem em residências adaptadas, a inserção de algumas práticas integrativas é comprometida. O ambiente não comporta circulação de pessoas e tampouco permite uma correta desinfecção para utilização rotativa de determinados locais, tendo em vista as superfícies irregulares (VIEIRA IC, et al., 2018).

Além disso, a organização dos profissionais foi uma dificuldade relatada pela própria equipe na promoção em saúde. Alguns deles disseram que muitas vezes estão em atividades de promoção da saúde e precisam interrompê-la devido a um atendimento de demanda espontânea, o que gera desorganização entre a equipe e desestímulo do grupo participante das atividades (SILVEIRA MR, et al., 2011).

Alguns autores elucidaram o processo de trabalho médico-centrado como um empecilho à implementação das PICs (SILVEIRA MR, et al., 2011). Um estudo realizado em 2011 em 10 unidades básicas de saúde na cidade de Belo Horizonte, capital do estado de Minas Gerais, revelou a tentativa de compartilhar entre a equipe de saúde a responsabilidade de promoção da saúde. Segundo os profissionais ouvidos, a discussão de propostas de melhoria ficava restrita a apenas um dia da semana e muitas vezes esse ponto de discussão não era colocado em pauta, perdendo lugar para casos mais complexos do cotidiano semanal (SILVEIRA RP e ROCHA CM, 2020).

Também foram relatadas dificuldades relacionadas à população, como resistência em dar crédito às Práticas e participar de atividades que geram resultados em um médio e longo prazo (CRUZ PLB e SAMPAIO SF, 2016). Além disso, nesse estudo os entrevistados médicos afirmaram não conhecer a homeopatia, e não dar crédito à técnica, e se recusaram a utiliza-la sem sequer ter conhecimento acerca delas (BARROS LCN, et al., 2014).

Os autores Losso LN e Freitas SFT (2017), desenvolveram um método de avaliação do grau de implantação das PICS na Atenção básica em municípios do estado de Santa Catarina. Dentre os 293 municípios analisados, a maior parte dos municípios que conseguiram o mais alto grau de avaliação eram municípios de até 5 mil habitantes. Os componentes avaliados foram: assistência, recursos humanos, recursos materiais e gestão. Uma dificuldade visualizada por esses autores, e diferenciada entre cidades maiores e menores, foi a gestão pública. Os municípios pequenos tiveram dificuldades em responder às ações que a Política Nacional de Atenção Básica (PNAB) estipula. Já para os municípios maiores, os quais possuem um apoiador institucional de gestão, apresentam as áreas da gestão de recursos em níveis de organização, instituições, política e financeiro bem articulados. Esse desafio traz à tona a necessidade de investimento nos pequenos municípios, pois, mesmo que tenha dificuldades na gestão, há grande possibilidade de implantação eficaz das PICs em cada um deles, segundo a avaliação dos pesquisadores.

\section{Propostas governamentais e privadas destinadas a adesão da Medicina Integrativa na atenção básica}

Entre as diretrizes contempladas pela PNPIC para os gestores municipais destacam-se: estabelecimento de instrumentos de gestão e indicadores de acompanhamento; promoção de articulação intersetorial para efetivação da política; elaboração de normas técnicas para inserção da PNPIC na saúde das redes municipais (SILVEIRA RP e ROCHA CM, 2020).

Como proposta de implantação eficaz das PNPIC no SUS o Fórum de Discussão Telessaúde Santa Catarina com parceria da Universidade Federal de Santa Catarina (UFSC) em 2018, segmentou o projeto em algumas fases que visam abranger a grandeza do sistema e alguns dos percalços supracitados. A estratégia do Fórum se baseou na criação de uma proposta, elaboração de plano de desenvolvimento e implantação, métricas para avaliação e acompanhamento das políticas, financiamento e até orientação para qualificação das equipes de saúde na atenção básica e no e-SUS (DRAGON LC, 2018).

Uma estratégia citada pelo MS foi a implantação em um município de 30 mil habitantes, onde os profissionais que já possuíam experiência com alguma PICs e também houve o incentivo dos profissionais 
interessados em conhecer a educação continuada promovida pelo Ambiente Virtual de Aprendizagem do Sistema Único de Saúde (AVASUS). Após a pactuação entre os profissionais e gestores, foi feito um mapeamento sobre perfil populacional: quantidade de crianças, idosos, pessoas em sofrimento mental, hipertensos, entre outras doenças (SILVEIRA MR, et al., 2011).

A análise das estruturas que existem nos estabelecimentos de saúde também é essencial para realização de atividades coletivas e individuais, além da identificação de insumos necessários para sua execução. Por fim, após recolher uma devolutiva da equipe de profissionais, elaboraram um projeto em conjunto contendo plano de ação com objetivos, responsáveis e cronograma das etapas (ALONSO SG, et al., 2019).

Já Dalmolin IS e Heidemann ITSB (2020), mostraram uma abordagem pedagógica para compreensão do meio social antes da implementação da PICS, explorando a qualificação de um grupo de pessoas que possuem algum interesse comum. Por meio da discussão sobre seus problemas e situações de vida, constroem uma percepção mais profunda de sua realidade. Desse modo, o centro da ação torna-se o usuário do SUS. O ciclo é dividido entre pesquisa temática; codificação e decodificação; revelação crítica. Na fase de pesquisa, há o descobrimento da linguagem usada e vocabulário local. Já na segunda, há a expansão do conhecimento cultural sobre a população selecionada. Na última fase, a interpretação dos dados colhidos e levantamento de hipóteses são feitas para buscar entender a realidade e possibilidade de trabalho.

Outra estratégia criada para implantação das PICs na atenção básica propõe como $1^{\text {a }}$ etapa a sensibilização dos gestores de saúde locais, profissionais da ESF e rede de apoio social. Fazem parte dessa rede a religião, comunidade, grupos de educação, sindicatos, partidos políticos, grupos de meio ambiente e defensores de direito da cidadania e coletivo. Nessa intervenção, os profissionais de saúde vão até esse grupo com objetivo de colher informações e criar um perfil daquela comunidade, com base em suas características (VIEIRA IC, et al., 2018).

As PICs ganharam força na década de 90, alcançando proporções mundiais. Através do estímulo da Organização Mundial de Saúde, tais práticas foram incentivadas e regulamentadas nos serviços de saúde (RUELA LO, et al., 2019). Ainda de acordo com Ruela LO, et al. (2019), a PNPIC foi crucial para a difusão dessas práticas, alcançando assim um público muito maior do que anteriormente. Os autores acreditam que apesar das dificuldades ainda existentes, as PIC estão cada vez mais acessíveis e fazem parte da vida de milhões de pessoas. Ainda refletindo sobre o cenário nacional, os autores ainda afirmamque acreditam no avanço das PIC no Brasil.

Por outro lado, Jales RD, et al. (2020), demonstraram uma visão mais receosa sobre o assunto relatando que o conhecimento sobre as Práticas Integrativas e Complementares e sobre a Política Nacional de Práticas Integrativas e Complementares ainda é limitado. De acordo com os autores, algumas práticas por parte dos governantes acabam por atrapalhar ou mesmo impedir a difusão das PICs pelo cenário nacional.

\section{CONSIDERAÇÕES FINAIS}

Fica evidente que a falta de infraestrutura na ABS dificulta a implementação da medicina complementar e integrativa no cotidiano funcional das unidades. Essa carência é explicada tanto pela falta de insumos quanto pela falta de incentivos governamentais à implementação das PICs. Em adição a isso, é de suma importância destacar que as escolas de ensino superior no Brasil raramente oferecem aos alunos da área da saúde o aprendizado sobre práticas integrativas, mesmo após a publicação da portaria em 2006 pelo MS. Nesse interim, essa revisão integrativa aponta os impactos benéficos da medicina integrativa na saúde pública, assim como relata os principais obstáculos da difusão dessas práticas no cenário político e social da hodiernidade brasileira.

\section{REFERÊNCIAS}

1. ALBUQUERQUE LVC, et al. Complementary and Alternative Medicine Teaching: Evaluation of theTeaching-Learning Process of Integrative Practices in Brazilian Medical Schools. Rev. bras. educ. med., 2019; 43(4): 109-116. 
2. ALMEIDA JRGS, et al. Avaliação do conhecimento de estudantes universitários sobre medicina alternativa. Revista baiana de saúde pública, 2016; 40(2): 289-297.

3. ALONSO SG, et al. Predictive, Personalized, Preventive and Participatory (4P) Medicine Applied to Telemedicine and eHealth in the Literature. Journal of Medical Systems, 2019; 43(5): e140.

4. BARROS LCN, et al. Práticas Integrativas e Complementares Na Atenção Primária à Saúde: Percepções Dos Gestores Dos Serviços. Esc. Anna Nery, 2020; 24(2): e20190081.

5. MINISTÉRIO DA SAÚDE. Manual de Implantação de Serviços de Práticas Integrativas e Complementares no SUS. Secretraia de Atenção à Saude, Departamento de Antenção Básica. 2018. Disponível em: http://189.28.128.100/dab/docs/portaldab/publicacoes/manual_implantacao_servicos_pics.pdf. Acessado em: 20 de outubro de 2021.

6. CRUZ PLB, SAMPAIO SF. As Práticas Terapêuticas Não Convencionais Nos Serviços de Saúde: Revisão Integrativa. Rev. APS, 2016; 19(3): 483-494.

7. DALMOLIN IS, HEIDEMANN ITSB. Integrative and complementary practices in Primary Care: unveiling health promotion. Rev. Latino-Am. Enfermagem, 2020; 28: e3277.

8. DAMASCENO CMD, et al. Avaliação do conhecimento de estudantes universitários sobre medicina alternativa. Revista baiana de saúde pública, 2016; 40(2): 289-297.

9. DRAGO, LC. Gestão da educação permanente e o cuidado de enfermagem e saúde na implementação da política nacional de práticas integrativas e complementares em Florianópolis. Tese de doutorado (Programa de PósGraduação em Enfermagem) - Universidade Federal de Santa Catarina, 2018; 218p.

10. FAQUETI A, TESSER CD. Utilização de Medicinas Alternativas e Complementares na atenção primária à saúde de Florianópolis/SC, Brasil: percepção de usuários. Ciência \& Saúde Coletiva, 2018; 23(8): 2621-2630.

11. HABIMORAD PHL, et al. Implementation of Brazil's National Policy on Complementary and Integrative Practices: strengths and weaknesses. Ciência \& Saúde Coletiva, 2020; 25(2): 395-405.

12. JALES RD, et al. Conhecimento e Implementação Das Práticas Integrativas e Complementares Pelos Enfermeiros Da Atenção Básica. Rev. Pesqui, 2020; 12: 808-813.

13. LOSSO LN, FREITAS SFT. Avaliação Do Grau Da Implantação Das Práticas Integrativas e Complementares Na Atenção Básica Em Santa Catarina, Brasil. SD, 2017; 41(3): 171-187.

14. NASCIMENTO MC, et al. Formação em práticas integrativas e complementares em saúde: desafios para as universidades públicas. Trab. Educ. Saúde, 2018; 16(2): 751-772.

15. RUELA LO, et al. Implementação, acesso e uso das práticas integrativas e complementares no Sistema Único de Saúde: revisão da literatura. Ciência \& Saúde Coletiva, 2019; 24(11): 4239-4250.

16. SANTOS LL, et al. Knowledge and acceptance of integrative and complementary practices by medical students. Rev. APS, 2018; 21(4): $646-666$.

17. SILVA GKF, et al. Política Nacional de Práticas Integrativas e Complementares: trajetória e desafios em 30 anos do SUS. Revista de Saúde Coletiva, 2020; 30(1): e300110.

18. SILVEIRA RP, ROCHA CMF. (De)construction of truths: an analysis about integrative and complementary practices in health. Saúde e Sociedade, 2020; 29(1): e180906.

19. SILVEIRA MR, et al. O Processo De Trabalho Das Equipes De Saúde Da Família: Implicações Para A Promoção Da Saúde. Rev. Min. Enferm, 2011; 15(2): 196-20.

20. VIEIRA IC, et al. Demand for health care in integrative and complementary practices by primary care users and associated factors. Rev. APS, 2018; 21(4): 551-569.

21. WORLD HEALTH ORGANIZATION (WHO). The Ottawa Charter for Health Promotion. Geneva: World Health Organization; 1986. Disponível em: https://www.who.int/teams/health-promotion/enhanced-wellbeing/first-globalconference. Acesso em 20 de Outubro de 2021. 